\section{Fraturas de fêmur em idosos no Brasil: análise espaço-temporal de 2008 a 2012}

\author{
Femoral fractures in elderly Brazilians: \\ a spatial and temporal analysis from 2008 to 2012
}

\author{
Las fracturas de fémur de ancianos en Brasil: \\ análisis espacio-temporal de 2008 a 2012
}

\author{
${ }^{1}$ Faculdade de Medicina de \\ Ribeirão Preto, Universidade \\ de São Paulo, Ribeirão Preto, \\ Brasil. \\ Correspondência \\ A. A. Nunes \\ Faculdade de Medicina de \\ Ribeirão Preto, Universidade \\ de São Paulo. \\ Av. Bandeirantes 3900 \\ Ribeirão Preto, SP \\ 14049-900, Brasil. \\ altacilio@fmrp.usp.br
}

\begin{abstract}
Fractures in the elderly ( $\geq 60$ years) have a major public health impact and take a heavy social and economic toll. This article aimed to describe spatial and time trends in femoral fractures among elderly men and women in all regions of Brazil. Bayesian descriptive analyses of spatial and time series were performed on data obtained from the Hospital Information System of the Brazilian Unified National Health System, using Poisson regression for femoral fractures in individuals 60 years of age or older from 2008 to 2012. There were more than 181,000 femoral fractures during this period, predominantly in women, with out important spatial correlations or temporal differences. Despite the lack of temporal and spatial correlations, the number of femoral fractures in elderly Brazilians was high, with heavy financial and social costs. Public health policies are urgently needed to control predisposing factors for femoral fractures in elderly Brazilians.
\end{abstract}

Femoral Fractures; Hip Fractures; Wounds and Injuries; Aged

\author{
Danilo Simoni Soares 1 \\ Luane Marques de Mello 1 \\ Anderson Soares da Silva 1 \\ Edson Zangiacomi Martinez 1 \\ Altacílio Aparecido Nunes 1
}

\section{Resumo}

Fraturas de fêmur em pessoas com idade igual ou superior a 60 anos (idosos) representam um grande impacto para a saúde pública, e estão associadas à elevada morbimortalidade e grandes custos socioeconômicos. Buscou-se descrever temporal e espacialmente os casos de fratura de fêmur em idosos de todas as regiões do país, por sexo, em um período de cinco anos. Foram realizadas descrições de série temporal e espacial bayesiana, baseadas em dados obtidos do Sistema de Informações Hospitalares do Sistema Único de Saúde (SIH-SUS), empregando modelo de regressão de Poisson, sobre os casos ocorridos entre os anos de 2008-2012. No período estudado ocorreram mais de 181 mil casos de fratura de fêmur, predominando o sexo feminino, sem correlações espaciais e diferenças temporais importantes. Apesar de não se observar predomínio de comportamento temporal e espacial, o número de casos de fratura de fêmur no Brasil é alto $e$ com grandes custos financeiros e sociais. Políticas públicas de saúde visando a controlar os fatores predisponentes para esse evento devem ser urgentemente implementadas.

Fraturas do Fêmur; Fraturas do Quadril; Ferimentos e Lesões; Idoso 


\section{Introdução}

Apesar de algumas variações geográficas quando à sua definição, para a Organização Mundial da Saúde (OMS) e o Ministério da Saúde do Brasil, a partir do marco legal da Política Nacional do Idoso e Estatuto do Idoso, biologicamente são considerados idosos todos com idade igual ou superior a 60 anos 1,2. O envelhecimento da população é um fenômeno de relevância mundial, estima-se que em 2050 existirão mais de 2 bilhões de pessoas com mais de 60 anos no mundo ${ }^{1}$, com projeções de 28 milhões de idosos no Brasil em 2020 2. Com o envelhecimento, observa-se a diminuição progressiva e fisiológica da reserva funcional dos indivíduos (senescência) que, em situações desfavoráveis de sobrecarga e estresse, pode levar à perda patológica da sua capacidade funcional (senilidade) 3, comprometendo suas habilidades físicas e mentais, além da independência na vida diária e capacidade de integração social 4.

O envelhecimento pode ser influenciado por fatores biológicos, sociais, econômicos, além de causas externas e doenças 5 . As quedas fazem parte do grupo de causas externas e estão associadas à diminuição significativa da capacidade funcional do idoso, e sua ocorrência tende a aumentar com a idade, chegando a $51 \%$ nos indivíduos com mais de 85 anos 6 . As quedas, em geral, se devem à marcha instável e perda do equilíbrio decorrente da insuficiência súbita dos mecanismos neuro-sensoriais e osteoarticulares envolvidos na manutenção da postura 7. As principais consequências das quedas em idosos são fraturas, aumento do risco de morte, o medo futuro de novas quedas levando à restrição de atividades, $o$ declínio da saúde global e o aumento do risco de institucionalização ${ }^{8}$.

Dentre as fraturas, as mais comuns são as de fêmur ${ }^{9}$ para as quais a osteoporose é um importante fator de risco 10 , justificando sua maior ocorrência entre as mulheres com idade igual ou superior a 60 anos 11,12. Os números relativos a esse problema refletem sua importância em algumas partes do mundo. Nos Estados Unidos, há registros de 0,8 caso/mil pessoas com idade $\geq$ 60 anos/anualmente, com gastos anuais aproximados de 10 bilhões de dólares 13 . No continente europeu os casos apresentam uma distribuição característica, com incidência mais elevada nos países do norte como Suécia, Dinamarca e Noruega, e menores na região do Mediterrâneo 14,15,16. Na América Latina, os coeficientes de incidência de fratura de fêmur, sobretudo em mulheres com idade superior a 50 anos, também apresentam variações entre os países, sendo de $0,45 \mathrm{caso} / \mathrm{mil}$ pessoas com idade $\geq 60 \mathrm{anos} / \mathrm{anu}$ - almente no Chile e 0,95 caso/mil pessoas com idade $\geq 60$ anos/anualmente na Venezuela 17 . As informações mais recentes sobre fraturas de fêmur em idosos no Brasil reportam cerca de 30 mil casos/ano, sendo responsáveis, em 2008, por 32.908 internações hospitalares no Sistema Único de Saúde (SUS), com um custo total de 58,6 milhões de reais 18 .

Nos últimos anos, as fraturas de fêmur têm merecido atenção das autoridades sanitárias brasileiras pelo seu evidente impacto na saúde dos idosos e por suas consequências para o setor público. Estudos atuais revelam que a expectativa de vida dos pacientes que sofrem esse tipo de fratura é reduzida em 15 a 20\%, com as taxas de mortalidade relacionadas a esse agravo variando de 15 a $50 \%$ no primeiro ano 13,19. Além disso, a incapacidade física total ou parcial após a fratura é outro grande problema, sendo que $50 \%$ dos pacientes tornam-se restritos ao leito ou à cadeira de rodas e, daqueles que conseguem retornar ao domicílio, 25 a 35\% passam a necessitar de cuidadores ou algum dispositivo para auxiliar a locomoção 14,20. Estudo recente avaliando a capacidade funcional e a qualidade de vida de idosos com história de fratura de fêmur um ano após o tratamento cirúrgico identificou dificuldade para deambular com necessidade de auxílio em $44,2 \%$, com menores chances de recuperação da marcha naqueles com idade igual ou superior a 80 anos 21 . Outro estudo, também avaliando idosos um ano após a fratura de fêmur, observou dependência parcial na realização das atividades da vida diária em 19,6\% deles e dependência total em 13,7\%, significando a existência de algum grau de dependência funcional em mais de $30 \%$ dos pacientes 4 .

Diante da importância e da escassez de estudos sobre o tema no Brasil, o presente trabalho tem por finalidade descrever a evolução temporal e espacial dos casos de fratura de fêmur na população de pessoas com idade igual ou superior a 60 anos em todas as regiões do país, por sexo, em um período de cinco anos.

\section{Métodos}

Trata-se de um estudo ecológico com componente de série temporal empregando-se análise bayesiana de distribuição espacial, com dados obtidos do Sistema de Informações Hospitalares do SUS (SIH-SUS), em que foram incluídos os casos de fratura de fêmur (sem discriminar a localização anatômica específica), portanto, abrangendo todos os códigos relativos a 10a revisão da Classificação Internacional de Doenças (CID-10) em pessoas com idade maior ou igual a 60 anos 
entre os anos de 2008 a 2012, sendo analisados de acordo com as Unidades da Federação (UF) do Brasil em relação ao sexo.

\section{Modelo estatístico}

Para a análise espacial das taxas de incidência de fraturas utilizou-se um mapeamento das razões de incidência padronizadas 22 (standardized incidence ratio, ou SIR, em inglês). A SIR é um método indireto de ajustamento de taxas por idade, que descreve em termos numéricos o quanto a incidência do evento de interesse em uma dada UF e em dado ano equivale à taxa encontrada no país como um todo. Para o sexo $s(\operatorname{com} s=1$ para o sexo feminino e $s=2$ para o masculino) e ano $t$ ( $t=1$ para 2008, $t=2$ para 2009, e assim sucessivamente) foram obtidos do SIH-SUS os números de fraturas ocorridas no Brasil em cada uma das seguintes faixas etárias: 60 a 64 anos $(f=1), 65$ a $69(f=2), 70$ a $74(f=3), 75$ a $79(f=4)$ e 80 anos e mais $(f=5)$. Esses números, divididos pela população brasileira para o correspondente sexo, ano e faixa etária, são as taxas de fraturas para o país, denotadas por $w(s, t, f)$.

O número esperado $E(p, s, t)$ de fraturas para a UF $p(p=1, \ldots, 27)$ durante o ano $t$ e o sexo $s$ é dado:

$E(p, s, t)=\sum_{f=1} w(s, t, f) \times m(p, s, t, f)$,

em que $m(p, s, t, f)$ é a população para a UF $p$, sexo $s$, ano $t$ e faixa etária $f$ (dados obtidos do Instituto Brasileiro de Geografia e Estatística - IBGE) 2. A SIR é dada por:

$\operatorname{SIR}(p, s, t)=\frac{Y(p, s, t)}{E(p, s, t)}$,

em que $Y(p, s, t)$ é o número observado de fraturas na UF $p$, sexo $s$ e ano $t$.

Assim, o modelo de regressão de Poisson considerado foi:

$Y(p, s, t) \mid \mu(p, s, t), E(p, s, t) \sim$ Poisson $(E(p, s, t) \times \mu(p, s, t))$, em que $\mu(p, s, t)$ é o parâmetro que representa a SIR, dado por:

$\mu(p, s, t)=\exp \left[\alpha_{0}+\alpha_{s p}+\omega(p, s, t)\right]$.

Nessa expressão, o parâmetro $\alpha 0$ é um intercepto constante e $\alpha_{s p}, s=1,2, p=1, \ldots, 27$, são efeitos aleatórios bivariados que capturam possíveis correlações espaciais entre as medidas de SIR considerando-se os efeitos de região de UF vizinhas. Assumiu-se que $\alpha_{s p}$ segue uma estrutura condicional autorregressiva intrínseca normal bivariada (MVCAR), cujos detalhes técnicos podem ser obtidos em Besag \& Kooperberg 23. Em adição, os efeitos $\omega(p, s, t)$ modelam a tendência longitudinal da SIR para cada UF e sexo, considerando um processo gaussiano multivariado com uma média de vetor $5 \times 1$, todos os componentes iguais a zero e função de covariância dada por:

$\operatorname{cov}\left[\omega(p, s, t), \omega\left(p, s, t^{*}\right)\right]=\theta_{s 1} \exp \left(-\theta_{s 2}\left|t-t^{*}\right|\right)$,

para $s=1,2, t=1, \ldots, 5$ e $t^{*}=1, \ldots, 5$ (detalhes técnicos são encontrados em Branscun et al. 24). Notar que essa expressão diz respeito à covariância entre os efeitos $\omega(p, s, t)$ e $\omega\left(p, s, t^{*}\right)$, sendo que $t$ e $t^{*}$ denotam dois anos quaisquer compreendidos no período estudado.

$\mathrm{Na}$ análise bayesiana 24 , foi especificada uma distribuição "flat" a priori para $\alpha_{0}$ e distribuições uniformes no intervalo $(0, K)$ para $\theta_{11}, \theta_{12}, \theta_{21} \mathrm{e}$ $\theta_{22}$, em que $K=50$. Entende-se como "flat" uma distribuição imprópria não informativa, ou seja, uma função constante para toda a extensão dos possíveis valores do parâmetro, mas a rigor não equivalente a uma distribuição de probabilidade. Uma análise de sensibilidade foi conduzida para investigar a influência de outros valores para $K$ no ajuste do modelo. Assumiram-se distribuições $a$ priori não informativas para todos os parâmetros do modelo. As distribuições condicionais $a$ posteriori para os parâmetros foram simuladas usando-se o método de Monte Carlo em cadeia de Markov (MCMC) por meio do software OpenBUGS (Medical Research Council, Biostatistics Unit, Cambridge, Reino Unido) 25. A estrutura espacial dos dados foi testada comparando-se modelos que incluem ou não os efeitos espaciais $\alpha_{s p}$ e pela observação dos índices de Moran 26 de correlação espacial. Na estimação desses índices, utilizou-se uma matriz de ponderações espaciais dada pelo critério da contiguidade, em que se consideraram elementos iguais a 1 para UF vizinhas e 0 caso contrário. Foram encontrados ainda valores- $p$ para os índices de Moran, utilizando-se a biblioteca ape do programa R (The R Foundation for Statistical Computing, Viena, Áustria; http:/ / www.r-project.org).

\section{Aspectos éticos}

Este estudo foi aprovado pelo Comitê de Ética em Pesquisa da Faculdade de Medicina de Ribeirão Preto (no 456/2011).

\section{Resultados}

Como pode ser observado na Tabela 1 , durante o período de estudos ocorreram mais de $181 \mathrm{mil}$ casos de fratura de fêmur em todo Brasil, considerando-se as cinco regiões do país.

Os casos aconteceram mais no sexo feminino $(67,5 \%)$, resultando em uma razão mulher/ 
Tabela 1

Distribuição do número absoluto de fraturas de fêmur em pessoas com idade $\geq 60$ anos, nas cinco regiões do Brasil e respectivas Unidades da Federação (UF) de 2008 a 2012, segundo os sexos.

\begin{tabular}{|c|c|c|c|c|c|c|c|c|c|c|c|c|c|c|c|}
\hline \multirow[t]{2}{*}{ Regiões (UF) } & \multicolumn{3}{|c|}{2008} & \multicolumn{3}{|c|}{2009} & \multicolumn{3}{|c|}{2010} & \multicolumn{3}{|c|}{2011} & \multicolumn{3}{|c|}{2012} \\
\hline & $M$ & $\mathbf{F}$ & Total & M & $\mathbf{F}$ & Total & $\mathrm{M}$ & $\mathbf{F}$ & Total & M & $\mathbf{F}$ & Total & M & $F$ & Total \\
\hline Norte & 459 & 660 & 1.119 & 475 & 787 & 1.262 & 409 & 662 & 1.071 & 543 & 812 & 1.355 & 615 & 910 & 1.525 \\
\hline $\mathrm{RO}$ & 57 & 52 & 109 & 73 & 86 & 159 & 82 & 94 & 176 & 55 & 54 & 109 & 47 & 47 & 94 \\
\hline$A C$ & 21 & 45 & 66 & 21 & 37 & 58 & 25 & 49 & 74 & 37 & 56 & 93 & 32 & 50 & 82 \\
\hline AM & 59 & 83 & 142 & 68 & 114 & 182 & 59 & 133 & 192 & 103 & 159 & 262 & 162 & 240 & 402 \\
\hline $\mathrm{RR}$ & 20 & 19 & 39 & 17 & 21 & 38 & 10 & 11 & 21 & 11 & 16 & 27 & 23 & 19 & 42 \\
\hline PA & 197 & 299 & 496 & 205 & 357 & 562 & 153 & 246 & 399 & 202 & 361 & 563 & 223 & 355 & 578 \\
\hline$A P$ & 20 & 37 & 57 & 24 & 43 & 67 & 13 & 18 & 31 & 24 & 27 & 51 & 29 & 44 & 73 \\
\hline TO & 85 & 125 & 210 & 67 & 129 & 196 & 67 & 111 & 178 & 111 & 139 & 250 & 99 & 155 & 254 \\
\hline Nordeste & 1.887 & 4.288 & 6.175 & 2.211 & 4.731 & 6.942 & 1.904 & 4.265 & 6.169 & 2.221 & 4.806 & 7.027 & 2.200 & 4.635 & 6.835 \\
\hline MA & 130 & 243 & 373 & 202 & 361 & 563 & 74 & 162 & 236 & 112 & 205 & 317 & 161 & 281 & 442 \\
\hline PI & 107 & 250 & 357 & 162 & 354 & 516 & 134 & 232 & 366 & 143 & 302 & 445 & 173 & 298 & 471 \\
\hline CE & 278 & 762 & 1040 & 393 & 1.018 & 1.411 & 319 & 785 & 1.104 & 302 & 760 & 1.062 & 289 & 685 & 974 \\
\hline $\mathrm{RN}$ & 160 & 366 & 526 & 155 & 376 & 531 & 148 & 426 & 574 & 179 & 441 & 620 & 188 & 399 & 587 \\
\hline PB & 201 & 524 & 725 & 261 & 587 & 848 & 230 & 540 & 770 & 211 & 488 & 699 & 221 & 510 & 731 \\
\hline PE & 301 & 725 & 1.026 & 271 & 537 & 808 & 280 & 661 & 941 & 385 & 887 & 1.272 & 390 & 881 & 1.271 \\
\hline $\mathrm{AL}$ & 120 & 287 & 407 & 132 & 272 & 404 & 61 & 144 & 205 & 114 & 226 & 340 & 112 & 293 & 405 \\
\hline SE & 91 & 174 & 265 & 102 & 202 & 304 & 121 & 263 & 384 & 130 & 273 & 403 & 83 & 210 & 293 \\
\hline BA & 499 & 958 & 1.457 & 533 & 1.024 & 1.557 & 537 & 1.052 & 1.589 & 645 & 1.224 & 1.869 & 583 & 1.078 & 1.661 \\
\hline Sudeste & 5.753 & 1.2230 & 17.983 & 6.470 & 13.280 & 19.750 & 6.461 & 13.362 & 19.823 & 6.554 & 14.296 & 20.850 & 6.833 & 14.344 & 21.177 \\
\hline MG & 1.621 & 3.279 & 4.900 & 1.808 & 3.576 & 5.384 & 1.797 & 3.618 & 5.415 & 1.810 & 3.817 & 5.627 & 1.929 & 3.874 & 5.803 \\
\hline ES & 202 & 397 & 599 & 209 & 365 & 574 & 166 & 335 & 501 & 212 & 450 & 662 & 226 & 477 & 703 \\
\hline RJ & 775 & 1.693 & 2.468 & 825 & 1.916 & 2.741 & 807 & 1.904 & 2.711 & 865 & 2.045 & 2.010 & 940 & 2.128 & 3.068 \\
\hline $\mathrm{SP}$ & 3.155 & 6861 & 10.016 & 3.628 & 7.423 & 11.051 & 3.691 & 7.505 & 11.196 & 3.667 & 7.984 & 11.651 & 3.738 & 7.865 & 11.603 \\
\hline Sul & 1.715 & 4.160 & 5.875 & 1.875 & 4.530 & 6.405 & 1.979 & 4.526 & 6.505 & 2.088 & 4.865 & 6.953 & 2.070 & 4.929 & 6.999 \\
\hline PR & 713 & 1.312 & 2.025 & 763 & 1.520 & 2.283 & 921 & 1.694 & 2.615 & 910 & 1.763 & 2.673 & 893 & 1.828 & 2.721 \\
\hline $\mathrm{SC}$ & 310 & 829 & 1.139 & 369 & 860 & 1.229 & 332 & 873 & 1.205 & 390 & 1.022 & 1.412 & 457 & 1.008 & 1.465 \\
\hline RS & 692 & 2.019 & 2.711 & 743 & 2.150 & 2.893 & 726 & 1.959 & 2.685 & 788 & 2.080 & 2.868 & 720 & 2.093 & 2.813 \\
\hline Centro-oeste & 711 & 1.087 & 1.798 & 828 & 1.248 & 2.076 & 786 & 1.266 & 2.052 & 755 & 1.179 & 2.034 & 845 & 1.374 & 2.219 \\
\hline MS & 169 & 202 & 371 & 190 & 309 & 499 & 183 & 319 & 502 & 225 & 334 & 559 & 209 & 383 & 592 \\
\hline $\mathrm{MT}$ & 160 & 233 & 393 & 202 & 238 & 440 & 127 & 201 & 328 & 126 & 161 & 387 & 167 & 221 & 388 \\
\hline GO & 279 & 426 & 705 & 301 & 454 & 755 & 310 & 459 & 769 & 271 & 411 & 682 & 308 & 529 & 837 \\
\hline DF & 103 & 226 & 329 & 135 & 247 & 382 & 166 & 287 & 453 & 133 & 273 & 406 & 161 & 241 & 402 \\
\hline Total & 10.525 & 22.425 & 32.950 & 11.859 & 24.576 & 36.435 & 11.539 & 24.081 & 35.620 & 12.161 & 25.958 & 38.219 & 12.563 & 26.192 & 38.755 \\
\hline
\end{tabular}

F: feminino; M: masculino.

UFs: AC: Acre; AL: Alagoas; AM: Amazonas; AP: Amapá; BA: Bahia; CE: Ceará; DF: Distrito Federal; ES: Espírito Santo; GO: Goiás; MA: Maranhão; MG: Minas Gerais; MS: Mato Grosso do Sul; MT: Mato Grosso; PA: Pará; PB: Paraíba; PE: Pernambuco; PI: Piauí; PR: Paraná; RJ: Rio de Janeiro; RN: Rio Grande do Norte; RO: Rondônia; RR: Roraima; RS: Rio Grande do Sul; SC: Santa Catarina; SE: Sergipe; SP: São Paulo; TO: Tocantins. Fonte: Departamento de Informática do SUS. Morbidade hospitalar do SUS por local de internação - notas técnicas. Origem dos dados e descrição das variáveis disponíveis para a tabulação. http://tabnet.datasus.gov.br/cgi/sih/midescr.htm (acessado em 30/Abr/2014). 
homem de 1,67/1, ou seja, a incidência de fraturas de fêmur em mulheres com idade $\geq 60$ anos, no período estudado, foi $67 \%$ superior à dos homens da mesma faixa de idade. A Região Sudeste do Brasil foi responsável por $54,7 \%$ de todos os casos e a Região Norte apenas por 3,5\%.

As taxas brutas de incidência de fraturas de fêmur distribuídas por sexo e respectiva relação entre os gêneros, considerando-se as regiões brasileiras, podem ser observadas na Tabela 2. Verifica-se que no período dos cinco anos estudados e considerando ambos os sexos, houve uma média geral de incidência de 1,80 fratura para cada mil idosos, com predomínio do sexo feminino cuja média geral de incidência foi de 2,04 fraturas/mil no período de 2008 a 2012.

As razões de incidência padronizadas (SIR) de cada UF para cada ano estudado, obtidas do modelo bayesiano, são descritas na Figura 1 para homens e na Figura 2 para mulheres. Para o sexo masculino, os índices de Moran estimados para os anos de 2008 a 2012 foram $0,02(p=0,68),-0,07$ $(p=0,83), 0,25$ ( $p=0,06), 0,15(p=0,23)$ e 0,15 ( $p=$ $0,22)$, respectivamente. Observamos uma maior correlação espacial a partir de 2010, embora os valores $p$ não evidenciem que estas medidas sejam significativamente maiores que zero.

Verificando-se as SIR relacionadas a fraturas de fêmur entre homens, pode-se notar que na maioria dos estados do Sudeste e Sul do Brasil elas ficaram acima de 1,1/mil homens com idade $\geq 60$ anos em todo o período avaliado, em contraste com os estados das regiões Norte e Nordeste que registraram SIR menores do que 1,0/ mil, em sua maioria.

Os índices de Moran estimados para os anos de 2008 a 2012, para o sexo feminino, foram $-0,06(p=0,87),-0,07$ ( $p=0,84), 0,31(p=0,02)$, $0,27$ ( $p=0,04)$ e $0,28(p=0,04)$, respectivamente, evidenciando que nos primeiros anos da série as medidas de SIR tenderam a distribuir-se de modo aleatório no país. A partir de 2010, os índices de Moran evidenciaram maior tendência a estarem mais agregados espacialmente do que estariam simplesmente pelo acaso.

De maneira muito semelhante ao observado entre os homens em relação as SIR relacionadas a fraturas de fêmur, nota-se que entre as mulheres com idade $\geq 60$ anos a ocorrência de fraturas incidiu com frequência ainda mais pronunciada do que a observada em homens, nos estados do Sudeste e Sul do Brasil, sendo que a partir de 2010, segundo os índices de Moran, esta tendência se mostrou ainda mais evidente.

\section{Discussão}

O presente trabalho avaliou os dados secundários registrados no SIH-SUS sobre a distribuição das fraturas de fêmur ocorridas no Brasil, no período de 2008 a 2012. Os resultados revelaram a ocorrência de mais de 181 mil casos de fraturas de fêmur em pessoas com idade $\geq 60$ anos no período de 2008 a 2012 em todo o país, representando uma média de 36.200 casos por ano, o que está de acordo com um estudo brasileiro realizado em 2008, ano em que os autores reportaram $30 \mathrm{mil}$ casos, gerando um custo de 58,6 milhões de reais em internações para os cofres públicos 18. Além da alta morbidade e mortalidade na população idosa, as fraturas de fêmur são responsáveis por significativa repercussão econômica e social de relevância não só para o Brasil, mas também

Tabela 2

Taxas brutas de incidência e razão de taxas brutas de incidência entre mulheres/homens relacionadas a fraturas de fêmur por mil pessoas com idade $\geq 60$ anos, por ano e regiões brasileiras de 2008 a 2012, Brasil.

\begin{tabular}{|c|c|c|c|c|c|c|c|c|c|c|c|c|c|c|c|c|c|c|c|c|}
\hline \multirow[t]{2}{*}{ Regiões } & \multicolumn{4}{|c|}{2008} & \multicolumn{4}{|c|}{2009} & \multicolumn{4}{|c|}{2010} & \multicolumn{4}{|c|}{2011} & \multicolumn{4}{|c|}{2012} \\
\hline & M & $F$ & F/M & Total & $\mathrm{M}$ & $F$ & $\mathrm{~F} / \mathrm{M}$ & Total & M & $\mathbf{F}$ & $\mathrm{F} / \mathrm{M}$ & Total & $\mathbf{M}$ & $F$ & $\mathrm{~F} / \mathrm{M}$ & Total & $\mathrm{M}$ & $F$ & $\mathrm{~F} / \mathrm{M}$ & Total \\
\hline Norte & 0,98 & 1,39 & 1,42 & 1,19 & 0,98 & 1,60 & 1,63 & 1,29 & 0,84 & 1,22 & 1,45 & 1,03 & 0,99 & 1,64 & 1,67 & 1,32 & 1,12 & 1,63 & 1,46 & 1,38 \\
\hline Nordeste & 0,87 & 1,60 & 1,84 & 1,27 & 0,99 & 1,72 & 1,73 & 1,39 & 0,78 & 1,42 & 1,82 & 1,10 & 0,64 & 1,53 & 2,39 & 1,08 & 0,63 & 1,52 & 2,41 & 1,08 \\
\hline Sudeste & 1,51 & 2,47 & 1,64 & 2,05 & 1,63 & 2,58 & 1,58 & 2,17 & 1,57 & 2,47 & 1,57 & 2,02 & 1,58 & 2,64 & 1,67 & 2,11 & 1,63 & 2,62 & 1,61 & 2,12 \\
\hline Sul & 1,25 & 2,44 & 1,95 & 1,91 & 1,32 & 2,56 & 1,95 & 2,01 & 1,36 & 2,48 & 1,82 & 1,92 & 1,43 & 2,68 & 1,87 & 2,06 & 1,40 & 2,67 & 1,91 & 2,03 \\
\hline Centro-oeste & 1,33 & 1,88 & 1,41 & 1,62 & 1,49 & 2,06 & 1,38 & 1,79 & 1,33 & 1,96 & 1,47 & 1,65 & 1,26 & 2,07 & 1,67 & 1,68 & 1,39 & 2,08 & 1,49 & 1,73 \\
\hline Total & 1,26 & 2,16 & 1,71 & 1,76 & 1,37 & 2,28 & 1,67 & 1,88 & 1,26 & 2,11 & 1,67 & 1,69 & 1,32 & 2,27 & 1,72 & 1,80 & 1,35 & 2,26 & 1,67 & 1,81 \\
\hline
\end{tabular}

F: feminino; M: masculino.

Fonte: Departamento de Informática do SUS. Morbidade hospitalar do SUS por local de internação - notas técnicas. Origem dos dados e descrição das variáveis disponíveis para a tabulação. http://tabnet.datasus.gov.br/cgi/sih/midescr.htm (acessado em 30/Abr/2014). 
1a) 2008

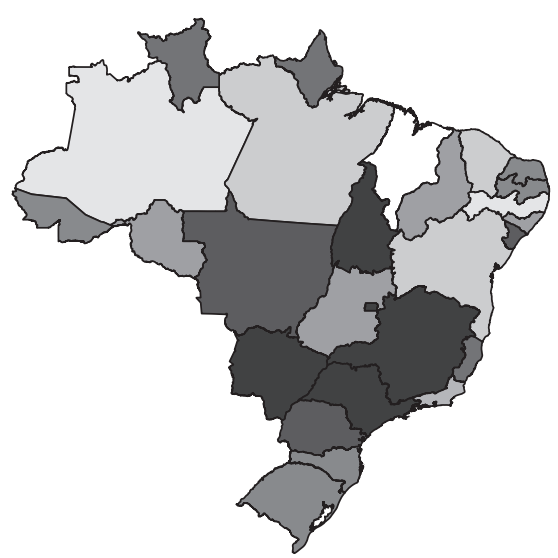

1d) 2011

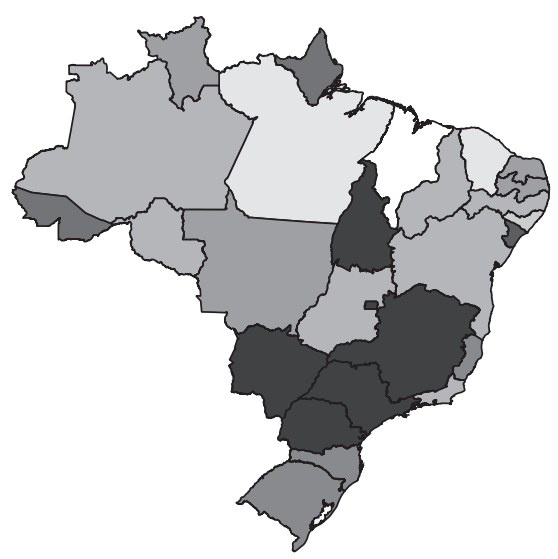

1b) 2009

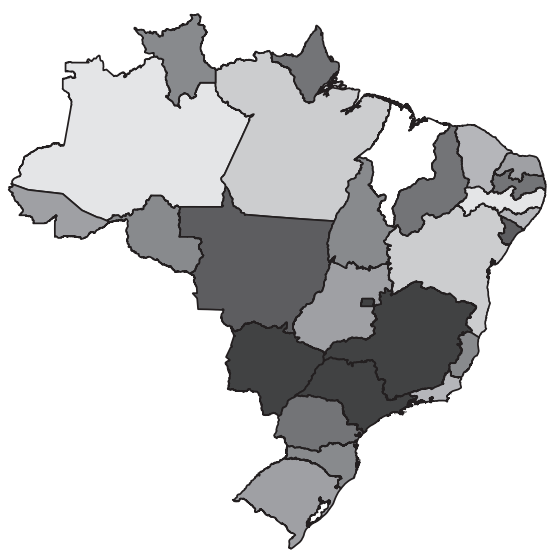

1e) 2012

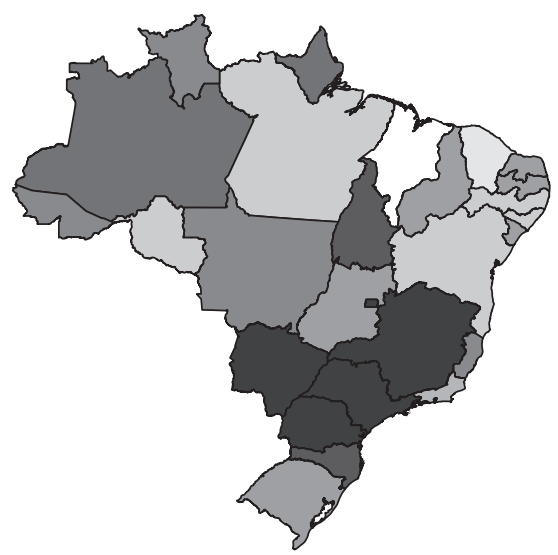

1c) 2010

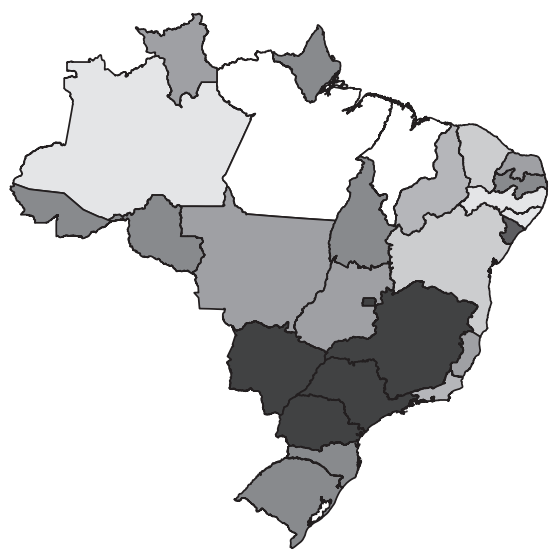

SIR

Menos de 0,50

$0,50-0,59$

$0,60-0,69$

$0,70-0,79$

$0,80-0,89$

$0,90-0,99$

1,00-1,09

$1,10-1,19$

1,20 ou mais

Fonte: Departamento de Informática do SUS. Informações de saúde (TABNET). Epidemiológicas e morbidade. http://www2.datasus.gov.br/DATASUS/index. php?area=0203\&VObj=http://tabnet.datasus.gov.br/cgi/deftohtm.exe?sih/cnv/ni (acessado em 30/Abr/2014).

para outros países. Há relatos de que nos Estados Unidos o custo médio das internações hospitalares por fraturas de fêmur seja de 26 mil dólares por episódio, sendo que o custo atribuído a esta condição durante toda a vida é de 81 mil dólares per capita 27 . Estima-se que o custo para cobrir as despesas com esse tipo de fratura a ser desembolsado pelos Estados Unidos em 2020 será de 16 bilhões de dólares 27,28 . No mundo, os custos diretos, indiretos, tangíveis e intangíveis associados às fraturas de fêmur em idosos são estimados em 131 bilhões de dólares anualmente 28 .
Dos mais de 181 mil casos de fraturas de fêmur identificados neste estudo, $67,5 \%$ ocorreram em mulheres, com uma razão mulher/homem de 1,67/1, confirmando os achados de outros trabalhos. Um estudo realizado na Inglaterra mostrou que as taxas médias anuais de fraturas de fêmur em homens foram de 0,55/mil e de 1,56/ mil em mulheres ${ }^{29}$, ou seja, uma razão mulher/ homem de 2,83/1. Em um estudo realizado com usuários do Medicare, nos Estados Unidos no período de 2000 a 2005, foi observado que as taxas de fraturas de fêmur em mulheres também fo- 


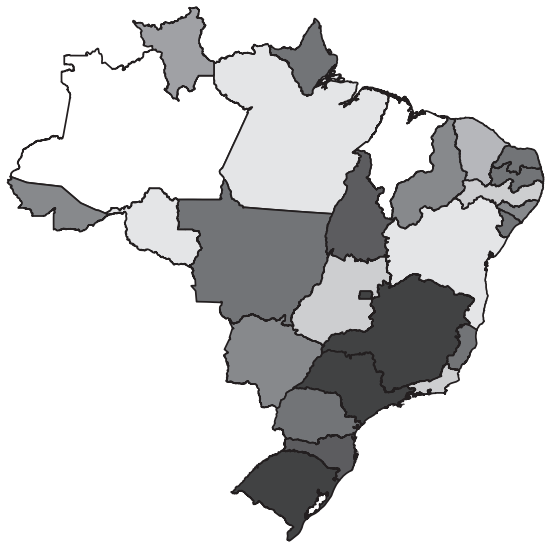

2d) 2011

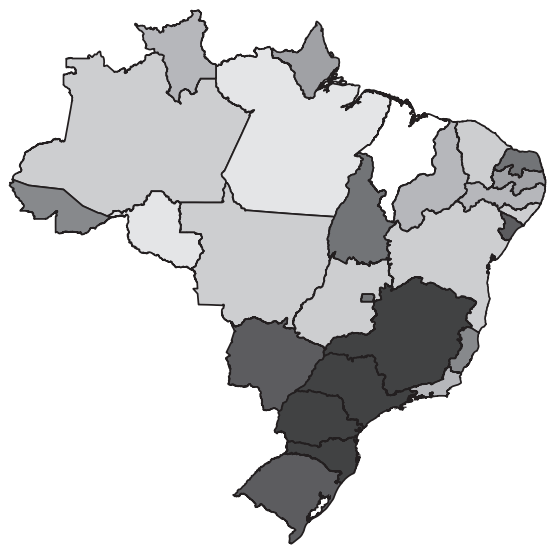

2b) 2009

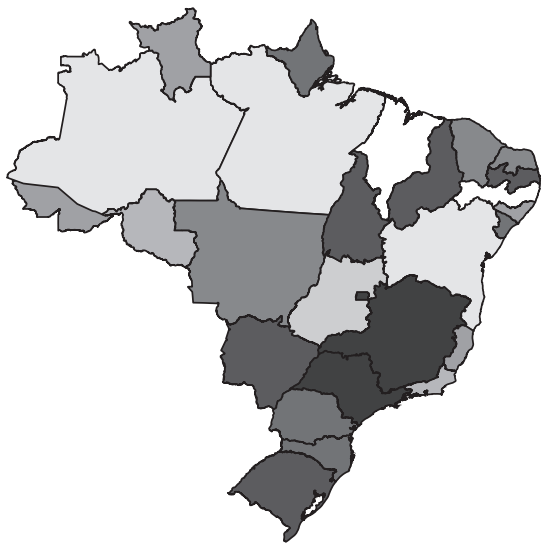

2e) 2012

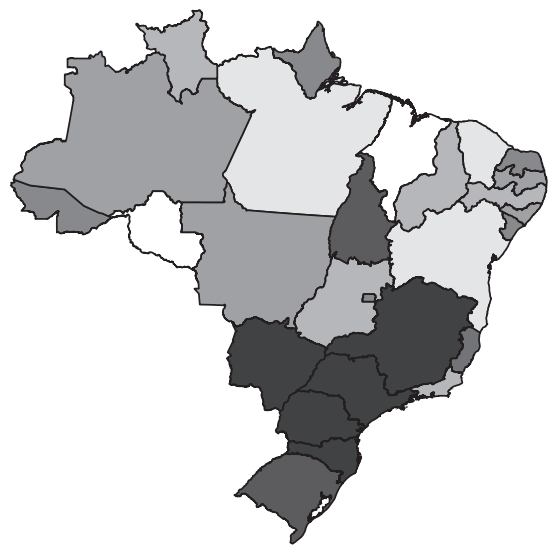

2c) 2010

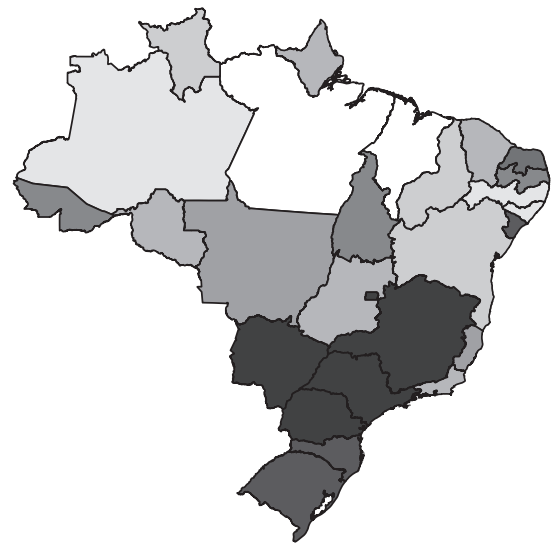

SIR

Menos de 0,50

$0,50-0,59$

$0,60-0,69$

$0,70-0,79$

$0,80-0,89$

$0,90-0,99$

1,00-1,09

$1,10-1,19$

Fonte: Departamento de Informática do SUS. Informações de saúde (TABNET). Epidemiológicas e morbidade. http://www2.datasus.gov.br/DATASUS/index. php?area=0203\&VObj=http://tabnet.datasus.gov.br/cgi/deftohtm.exe?sih/cnv/ni (acessado em 30/Abr/2014).

ram maiores do que nos homens 30 . Um estudo realizado na Coreia do Sul mostrou que no ano de $2003,70,2 \%$ das fraturas de fêmur ocorreram no sexo feminino e $29,8 \%$ no masculino 31 . Em outro estudo realizado na Noruega, com pacientes internados por fraturas de fêmur em quatro hospitais nos anos de 2004 e 2005, 71,5\% eram do sexo feminino e $28,5 \%$ do masculino 32 . Outra pesquisa realizada no norte da Índia mostrou que a incidência de fraturas de fêmur aumenta progressivamente com a idade, acometendo tanto homens quanto mulheres, mas, mesmo assim, as incidências de fraturas de fêmur na população com idade acima de 50 anos foram de 1,59/mil para mulheres e 1,05/mil para homens 33 .

Essa evidente suscetibilidade do gênero feminino chama a atenção para o problema da osteoporose, que é uma doença sistêmico-progressiva caracterizada pela diminuição da massa óssea e deterioração da microarquitetura, levando à fragilidade do osso e aumentando o risco de fraturas 34 . Sua prevalência tem aumentado em função do crescimento da população idosa e das mudanças no comportamento humano, tais 
como diminuição da ingestão de cálcio, sedentarismo, alcoolismo e tabagismo ${ }^{34}$. A osteoporose é o principal fator de risco para fraturas ósseas em idosos, especialmente as que acometem ossos longos, como o fêmur, tanto as ocasionadas por traumatismos quanto as de ocorrência espontânea 10. Segundo Pinto Neto et al. 35, 90\% das fraturas de fêmur e quadril na osteoporose ocorrem em razão de quedas, e o restante em consequência de movimentos de torção do fêmur com queda consequente. Evitando-se a queda, evitaremos $90 \%$ dessas fraturas.

A análise espacial realizada neste estudo sobre a ocorrência das fraturas de fêmur na população idosa brasileira identificou maior incidência na Região Sudeste, com 54,7\% de todos os casos. A Região Norte foi a região com a menor incidência, com apenas 3,5\% dos casos. Embora metodologicamente o estudo não permita afirmar as razões para e sobre essas diferenças, este foi um achado bastante interessante que levanta a possibilidade de que possíveis disparidades intrínsecas e extrínsecas, relacionadas a aspectos geográficos e climáticos, além de variações nos hábitos alimentares e até mesmo diferentes ações em saúde, instituídas de forma regionalizadas, poderiam influenciar a ocorrência das fraturas de fêmur e/ou de seus fatores de risco, como por exemplo, a osteoporose. Outros autores também relataram diferenças espaciais nas ocorrências de fraturas de fêmur, como Taylor et al. 30 , ao observarem que a taxa de fraturas de fêmur nos Estados Unidos é maior na Região Sul do que nas demais regiões, dado atribuído à variação geográfica verdadeira na incidência de fraturas ou a diferenças na sua detecção. Estudo realizado por Lalmohamed et al. 36 encontrou maior incidência de fraturas de fêmur nos países europeus do que na China, México e países na área do Mediterrâneo. Outro trabalho, realizado na Noruega, mostrou que a incidência de fraturas de fêmur em mulheres com mais de 50 anos é significativamente menor no sul do país do que na região de Oslo, a capital. Os autores justificam essa diferença geográfica às medidas farmacológicas e não farmacológicas destinadas ao con- trole da osteoporose que podem diferir entre as regiões 32 .

Em uma ampla, interessante e qualificada revisão sistemática recente 37 , os autores ao estudarem a incidência de fraturas de fêmur em 63 países classificaram-na em três grupos: alto, moderado e baixo risco para fraturas de fêmur, segundo os coeficientes de incidência, sendo que o Brasil foi categorizado como de baixo risco, e países desenvolvidos como Dinamarca, Suécia, Noruega, Suíça, entre outros, além de alguns em desenvolvimento como Argentina e Turquia, apresentaram altas taxas de incidência. Tais importantes e discrepantes diferenças, segundo os pesquisadores, poderiam ser explicadas mais por deficiências de registros ou composição da população do que por outras razões esperadas e possíveis como fatores socioeconômicos e biológicos, entre outros.

Um aspecto importante a ser aqui considerado, reside no fato de que neste estudo os dados coletados são referentes apenas a registros de hospitais essencialmente públicos ou conveniados ao SUS (prestadores de serviço), e assim, o número de fraturas de fêmur em pessoas idosas deve ser ainda maior do que o encontrado.

\section{Conclusão}

No presente estudo, apesar de não ter sido observado o predomínio de comportamento temporal e pouca correlação espacial associada à incidência da doença, o número de casos de fraturas de fêmur em idosos no Brasil a exemplo de outros países é alto, atingindo predominantemente mulheres, consequentemente com grandes custos financeiros e sociais. Políticas públicas de saúde visando a controlar os fatores predisponentes para esse evento devem ser urgentemente implementadas, especialmente se considerarmos que as estimativas preveem aumento significativo da população idosa nos próximos anos e consequentemente as doenças e fatores de risco relacionados à idade. 


\section{Resumen}

Las fracturas en personas mayores de 60 años (de edad avanzada) representan un impacto mayor de salud pública, se asocian con alta morbilidad y mortalidad y costos sociales y económicos importantes. El estudio trató de describir la evolución temporal y espacial de los casos de fractura de fémur en personas de edad $\geq 60$ años en todas las regiones del país, por sexo. Se realizaron descripciones de serie temporales y espaciales, mediante el método bayesiano, basadas en los datos obtenidos por el Sistema de Información Hospitalaria del Sistema Único de Salud (SIH-SUS), empleando el modelo de regresión de Poisson, sobre los casos ocurridos entre los años 2008-2012. En el periodo se produjeron más de 181.000 casos de fractura de fémur, predominantemente femenino, sin correlaciones importantes diferencias espaciales y temporales. Aunque no observamos ningún predominio del comportamiento temporal y espacial, el número de casos de fractura de fémur es alto con grandes costos financieros y sociales. Las políticas de salud públicas, destinadas a controlar los factores que predisponen para este hecho, deben aplicarse urgentemente.

Fracturas del Fémur; Fracturas de Cadera; Heridas y Traumatismos; Anciano

\section{Colaboradores}

D. S. Soares, L. M. Mello e A. S. Silva participaram da concepção da ideia, coleta de dados, redação e revisão do artigo e aprovação final da versão submetida. E. Z. Martinez e A. A. Nunes colaboraram na concepção da ideia, coleta de dados, análise e interpretação dos dados, redação e revisão do artigo e aprovação final da versão submetida.

\section{Referências}

1. Departamento de Atenção Básica, Secretaria de Atenção à Saúde, Ministério da Saúde. Envelhecimento e saúde da pessoa idosa. Brasília: Ministério da Saúde; 2006.

2. Instituto Brasileiro de Geografia e Estatística. Projeção da população do Brasil por sexo e idade, 1980-2050. Revisão 2008. Rio de Janeiro: Instituto Brasileiro de Geografia e Estatística; 2008. (Estudos e Pesquisas. Informação Demográfica e Socioeconômica, 24).

3. Aguiar CF, Assis M. Perfil de mulheres idosas segundo a ocorrência de quedas: estudo de demanda no Núcleo de Atenção ao Idoso da UnATI/UERJ. Rev Bras Geriatr Gerontol 2009; 12:9-20.

4. Cunha U, Veado MAC. Fratura da extremidade proximal do fêmur em idosos: independência funcional e mortalidade em um ano. Rev Bras Ortop 2006; 41:195-9.

5. Perracini MR, Ramos LR. Fatores associados a quedas em uma coorte de idosos residentes na comunidade. Rev Saúde Pública 2002; 36:709-16.

6. Rocha MA, Carvalho WS, Zanqueta C, Lemos SC. Estudo epidemiológico retrospectivo das fraturas do fêmur proximal tratados no hospital escola da Faculdade de Medicina do Triângulo Mineiro. Rev Bras Ortop 2001; 36:311-5.

7. Pereira SRM, Buksman S, Perracini M, Py L, Barreto KML, Leite VMM. Quedas em idosos. Rio de Janeiro: Sociedade Brasileira de Geriatria e Gerontologia; 2001.

8. Fabricio SCC, Rodrigues RAP, Costa Junior ML Causas e conseqüências de quedas de idosos atendidos em hospital público. Rev Saúde Pública 2004; 38:93-9.

9. Wei TS, Hu CH, Wang SH, Hwang KL. Fall characteristics, functional mobility and bone mineral density as risk factors of hip fracture in the community-dwelling ambulatory elderly. Osteoporos Int 2001; 12:1050-5.

10. Dore N, Kennedy C, Fisher P, Dolovich L, Farrauto L, Papaioannou A. Improving care after hip fracture: The fracture? Think osteoporosis (FTOP) program. BMC Geriatr 2013; 13:130.

11. Rocha MA, Carvalho WS, Zanqueta C, Lemos SC. Estudo epidemiológico retrospectivo das fraturas do fêmur proximal tratados no hospital escola da Faculdade de Medicina do Triângulo Mineiro. Rev Bras Ortop 2001; 36:311-5.

12. Muniz CF, Arnaut AC, Yoshida M, Trelha CS. Caracterização dos idosos com fratura de fêmur proximal atendidos em hospital escola público. Revista Espaço para a Saúde 2007; 8:33-8. 
13. Cummings SR, Kelsey JL, Nevitt MC, O’Dowd KJ. Epidemiology of osteoporosis and osteoporotic fractures. Epidemiol Rev 1985; 7:178-208.

14. Agnusdei D, Camporeale A, Gerardi D, Rossi S, Bocchi L, Gennari C. Trends in the incidence of hip fracture in Siena, Italy, from 1980-1991. Bone 1993; 14:31-4.

15. Lofthus CM, Osnes EK, Falch JA, Kaastad TS, Kristiansen IE, Nordsletten L, et al. Epidemiology of hip fractures in Oslo, Norway. Bone 2001; 29:413-8.

16. Bacon WE, Maggi S, Looker A, Harris T, Nair CR, Giaconi J, et al. International comparison of hip fracture rates in 1988-89. Osteoporos Int 1996; 6:69-75.

17. Mautalen C, Pimarino H. Epidemiology of osteoporosis in South America. Osteoporos Int 1997; 7 Suppl 3:S73-7.

18. Cunha PTS, Artifon AN, Lima DP, Marques WV, Rahal MA, Ribeiro RR. Fratura de quadril em idosos: tempo de abordagem cirúrgica e sua associação quanto a delirium e infecção. Acta Ortop Bras 2008; 16:173-6.

19. Haentjens P, Magaziner J, Colon-Emeric CS, Vanderschueren D, Milisen K, Velkeniers B, et al. Meta-analysis: excess mortality after hip fracture among older women and men. Ann Intern Med 2010; 152:380-90.

20. Alvarez-Nebreda ML, Jiménez AB, Rodríguez P, Serra JA. Epidemiology of hip fracture in the elderly in Spain. Bone 2008; 42:278-85.

21. Guimarães FAM, Lima RR, Souza ACS, Livani B, Belangero WD. Avaliação da qualidade de vida em pacientes idosos um ano após o tratamento cirúrgico de fraturas transtrocanterianas do fêmur. Rev Bras Ortop 2011; 46 Suppl 1:48-54.

22. Silva GL, Dean C. Modelling and analysis of disease incidence rates by age-groups over regions. Lisboa: Centro de Estatística e Aplicações da Universidade; 2012. (Notas e Comunicações, 19-2012).

23. Besag J, Kooperberg CL. On conditional and intrinsic autoregressions. Biometrika 1995; 82:733-46.

24. Branscun AJ, Perez AM, Johnson WO, Thurmond MC. Bayesian spatiotemporal analysis of foot-andmouth disease data from the Republic of Turkey. Epidemiol Infect 2008; 136:833-42.

25. Lunn DJ, Thomas A, Best N, Spiegelhalter D. WinBUGS - a bayesian modelling framework: concepts, structure, and extensibility. Stat Comput 2000; 10:325-37.
26. Kelsall JE, Wakefield JC. Modeling spatial variation in disease risk: a geostatistical approach. J Am Stat Assoc 2002; 97:692-701.

27. Ensrud KE. Epidemiology of fracture risk with advancing age. J Gerontol A Biol Sci Med Sci 2013; 68:1236-42.

28. Bonafede M, Espindle D, Bower AG. The direct and indirect costs of long bone fractures in a working age US population. J Med Econ 2013; 16:169-78.

29. Wu TY, Jen MH, Garrafa A, Liaw CK, Aylin P, Majeed A. Admission rates and in-hospital mortality for hip fractures in England 1998 to 2009: time trends study. J Public Health (Oxf) 2011; 33:284-91.

30. Taylor AJ, Gary LC, Arora T, Becker DJ, Curtis JR, Kilgore ML, et al. Clinical and demographic factors associated with fractures among older Americans. Osteoporos Int 2011; 22:1263-74.

31. Kang HY, Yang KH, Nam YK, Lua SH, Choi WJ, Ryong $\mathrm{KD}$, et al. Incidence and mortality of hip fracture among the elderly population in South Korea: a population-based study using the national health insurance claims data. BMC Public Health 2010; 10:230.

32. Diamantopoulos AP, Hoff M, Skoie M, Hochberg M, Haugeberg G. Short- and long-term mortality in males and females with fragility hip fracture in Norway: a population-based study. Clin Interv Aging 2013; 8:817-23.

33. Dhanwal DK, Siwach R, Dixit V, Mithal A, Jameson $\mathrm{K}$, Cooper C. Incidence of hip fracture in Rohtak district, North India. Arch Osteoporos 2013; 8:135.

34. Gali JC. Osteoporose. Acta Ortop Bras 2001; 9: 53-62.

35. Pinto Neto AM, Soares A, Urbanetz AA, Souza ACA, Ferrari AEM, Amaral B, et al. Consenso brasileiro de osteoporose. Rev Bras Reumatol 2002; 42: 343-54.

36. Lalmohamed A, Welsing PM, Lems WF, Jacobs JW, Kanis JA, Johansson H, et al. Calibration of Frax ${ }^{\circledR}$ 3.1 to the Dutch population with data on the epidemiology of hip fractures. Osteoporos Int 2012; 23:861-9.

37. Kanis JA, Odén A, McCloskey EV, Johansson H, Wahl DA, Cooper C, et al. A systematic review of hip fracture incidence and probability of fracture worldwide. Osteoporos Int 2012; 23:2239-56.

Recebido em 27/Dez/2013

Versão final reapresentada em 30/Abr/2014 Aprovado em 24/Jun/2014 